BI-TP 2003/20

\title{
Conditions for Confinement and Freeze-Out
}

\author{
V. Magas ${ }^{a}$ and H. Satz ${ }^{a, b}$ \\ a: Centro de Fisíca das Interacções Fundamentais (CFIF) \\ Instituto Superior Técnico, Av. Rovisco Pais, P-1049-001 Lisbon, Portugal \\ b: Fakultät fur Physik, Universität Bielefeld \\ Postfach 100 131, D-33501 Bielefeld, Germany
}

\begin{abstract}
:
Matter implies the existence of a large-scale connected cluster of a uniform nature. The appearance of such clusters as function of hadron density is specified by percolation theory. We can therefore formulate the freeze-out of interacting hadronic matter in terms of the percolation of hadronic clusters. The resulting freeze-out condition as function of temperature and baryo-chemical potential interpolates between resonance gas behaviour at low baryon density and repulsive nucleonic matter at low temperature, and it agrees well with data.
\end{abstract}

Consider a hot quark-gluon plasma in thermodynamic equilibrium, specified in terms of temperature $T$ and baryochemical potential $\mu$. Reducing the temperature of this medium at constant $\mu$ eventually brings it to the confinement transition at $T_{c}, \mu\left(T_{c}\right)$. Below this temperature, the system consists of interacting hadrons. Further cooling finally leads to freeze-out, at $T_{f}, \mu\left(T_{f}\right)$; beyond this point, we have non-interacting hadrons. The resulting two dividing lines in the $T-\mu$ plane are schematically shown in Fig. 1.

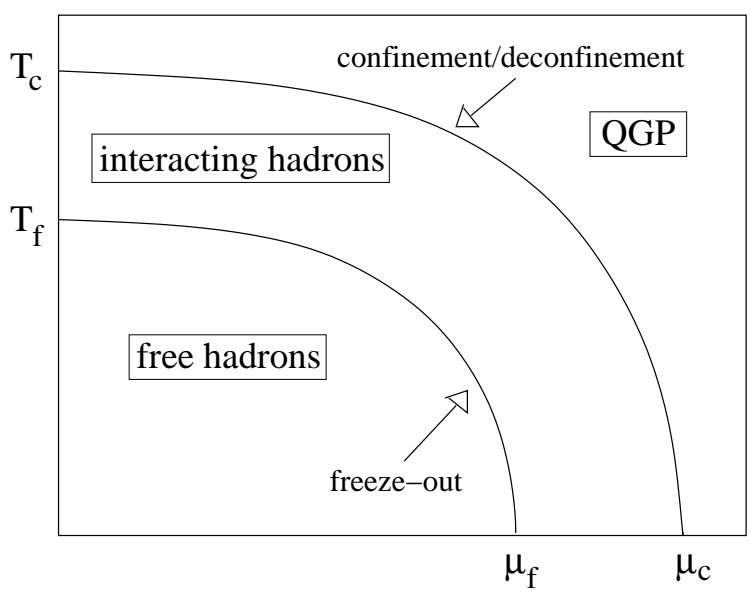

Figure 1: States of matter in QCD - general view 
While the line separating the deconfined QGP state from the confined hadronic medium is calculable in lattice QCD at finite temperature and density (although so far with considerable problems at large $\mu$ and low $T$ ), the freeze-out curve is less well-defined theoretically as well as experimentally. The aim of the present paper is to address how freeze-out can be specified conceptually.

In the two limiting cases of hot hadronic matter of vanishing overall baryon density and cold nuclear matter at vanishing temperature, we can make use of some specific aspects of the relevant dynamics. The confinement transition leads to an interacting hadronic medium. Now it is known that if the interactions between the constituents of such a medium are dominated by resonance formation and decay, the system can be treated as an ideal gas of all possible resonances [1, 2]. In our context this means that if and when the interacting hadronic medium is resonance dominated, freeze-out effectively occurs at the point of confinement. In particular, the relative abundances of the different species of hadrons and hadronic resonances are in this case determined at the transition from QGP to hadronic matter.

For systems of vanishing or low baryon density, this appears quite well supported. On a theoretical level, it is in fact claimed that resonance formation dominates the interaction between hadrons, perhaps most clearly in the dual resonance model [3]. Experimentally, an analysis of the species abundances indicate that these are indeed determined by a single freeze-out temperature, $T_{f} \simeq 175 \mathrm{MeV}$, obtained $e^{+} e^{-}$annihilation, $p-p$ and $p-\bar{p}$ interactions as well as in heavy ion collisions [4]. This temperature is moreover completely in accord with that found for the confinement transition in finite temperature lattice QCD [5].

On the other extreme, for dense nuclear matter at low temperature, the situation is quite different. The interaction between two nucleons does not lead to resonance formation; instead, it is dominated by Fermi statistics and baryon repulsion. In particular, at $T=0$, freeze-out must occur at a density equal to that of normal nuclear matter, $n_{0} \simeq 0.17 \mathrm{fm}^{-3}$. If we consider a system of non-interacting nucleons at $T=0$, the corresponding value of the baryochemical potential is obtained from

$$
n_{0}(T=0, \mu)=\frac{2}{3 \pi^{2}}\left(\mu^{2}-m^{2}\right)^{3 / 2}=0.17 \mathrm{fm}^{3}
$$

and found to be $\mu_{0} \simeq 0.979 \mathrm{GeV}$. Note that for $T=0$ and this value of $\mu_{f}$, the system consists only of nucleons. The threshold for the occurrence of baryon resonances is $\mu=$ $1.23 \mathrm{GeV}$, corresponding to $4.2 \mathrm{fm}^{-3}$ or about 25 times normal nuclear density. Hence interacting cold nuclear matter very likely contains only nucleons over the entire range from freeze-out at $\mu_{f}=\mu_{0} \mathrm{GeV}$ to the deconfinement value $\mu_{c}$, where it becomes cold quark matter. The freeze-out value of $\mu$ thus obtained is, however, in principle somewhat model-dependent. We have here assumed the repulsion to be totally given by Fermi statistics; there could well be additional repulsion beyond this. Using hard core repulsion with a core volume $V_{0}$ [6], Eq. 1] is replaced by

$$
n_{V}(T=0, \mu)=\frac{n_{0}(T=0, \mu)}{1+n_{0}(T=0, \mu) V_{0}}=0.17 \mathrm{fm}^{3} .
$$

The result is a freeze-out at slightly higher values of $\mu$. To illustrate, for $V_{0}=(4 \pi / 3) R^{3}$ and a nucleon radius of $0.8 \mathrm{fm}$, freeze-out occurs at $\mu_{V} \simeq 0.992 \mathrm{GeV}$. Such a hard core is 
presumably too large; reducing $R$ will reduce the resulting $\mu_{V}$, so that the physical value falls most likely between the values of $\mu_{0}$ and $\mu_{V}$.

Empirically, the determination of freeze-out parameters through the relative abundance of hadron species so far appears to be the only unambiguous approach to the problem. We therefore adopt this "chemical" freeze-out definition in the remainder of the paper. As an immediate consequence, we have to revise Fig. 1. At $\mu=0, T_{f}=T_{c}$, while at $T=0, \mu_{f} \simeq \mu_{0}<\mu_{c}$, as shown in Fig. 2. From what was said above, it is clear that for very low temperatures, specifying freeze-out through species abundances becomes problematic, since near $T=0$, the system contains only nucleons. This has to be kept in mind when studying cool baryon-rich media.

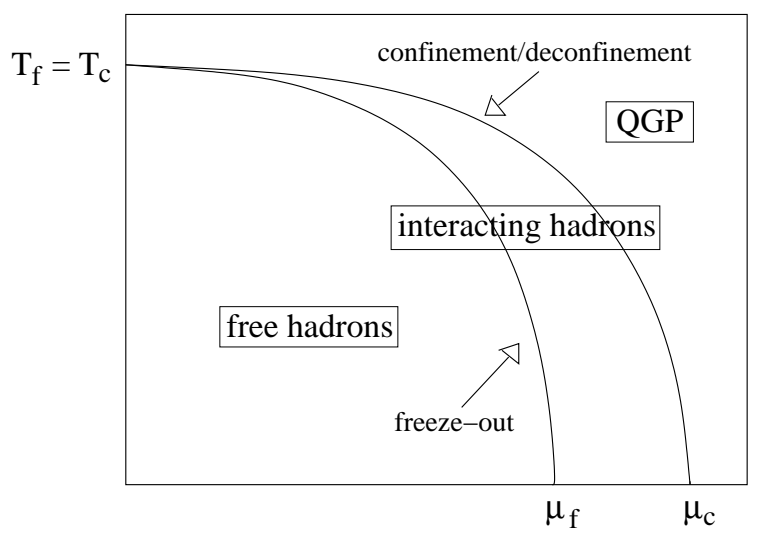

Figure 2: States of matter in QCD, including resonance gas effects

Looking at Fig. 2] we note that we have so far identified only $T_{f}=T_{c}$ at $\mu=0$ and $\mu_{f}$ at $T=0$. To determine the entire freeze-out curve in the $T-\mu$ plane, we have to know how the contributions from non-resonant baryon interactions modify the ideal resonance gas picture, in order to specify how the freeze-out curve departs from the confinement curve. In other words, we have to identify a freeze-out condition.

A very successful phenomenological parametrization [7] of the available heavy ion data is obtained by requiring the average hadron energy per average hadron number to be constant at freeze-out,

$$
\frac{\langle E\rangle}{\langle N\rangle} \simeq 1 \mathrm{GeV}
$$

While this parametrization accounts well for all data from SIS to RHIC experiments, it is clear that it encounters difficulties for sufficiently low temperature. At $T=0$, it leads to freeze-out at $\mu \simeq 1.039 \mathrm{GeV}$. With Fermi repulsion only, this corresponds to $n_{f} \simeq$ $0.73 \mathrm{fm}^{-3} \simeq 4.6 n_{0}$, with the hard core repulsion used above it gives $n_{f} \simeq 0.285 \mathrm{fm}^{-3} \simeq$ $1.7 n_{0}$. Both values would exclude the existence of normal nuclear matter, for which $\langle E\rangle /\langle N\rangle$ is below $1 \mathrm{GeV}$. Since it is also not known what features of the underlying physics could lead to Eq. (12) as freeze-out condition, it seems worthwhile to study possible freeze-out mechanisms and attempt to find a consistent description for the entire $T-\mu$ plane. 
Matter implies the existence of a large-scale interconnected medium of uniform nature. When such a system breaks up into fragments much smaller than the size of the volume in which it is contained, it has undergone a change of state. One possible way to define freeze-out is thus geometric: it occurs at the point at which the size of the largest hadronic clusters falls below the size of the given overall spatial volume. This point is determined in percolation theory [8] and for three space dimenensions becomes

$$
n_{f}=\frac{0.34}{V_{h}},
$$

where $n=N / V$ specifies the hadron density, with $N$ hadrons in the overall volume $V$. The volume of an individual hadron is denoted by $V_{h}=(4 \pi / 3) r_{h}^{3}$, and hadrons are allowed to overlap; thus $V_{h}$ introduces the short range nature $\left(\sim r_{h}\right)$ of hadronic forces [9].

When the hadron density has reached the percolation point $n=n_{f}$, the largest clusters reach the size of the overall volume. This, however, does not imply that they fill the volume. In fact, it is known that at the percolation point, still $\exp \{-0.34\} \simeq 71 \%$ remain empty space. Hence the vacuum, measured in terms of the hadronic scale $r_{h}$, also forms percolating clusters. We can therefore ask for what density vacuum percolation stops and only the strongly interacting medium spans the entire space. This occurs for

$$
n_{c}=\frac{1.24}{V_{h}},
$$

obtained in an analogous way as Eq. 1. For densities above $n=n_{c}$, any large-scale vacuum has disappeared. Since the disappearence of the physical vacuum is a basic feature of deconfinement, it seems natural to relate this threshold to the confinement/deconfinement transition.

Hence, on a purely geometric basis in terms of connected clusters, we have two thresholds: at $n=n_{f}$, there appear clusters of the size of the overall spatial volume, and at $n=n_{c}$, the vacuum disappears as a connected medium [9]. Let us see if and how they be related to the known freeze-out points at $T=0$ and at $\mu=0$.

At $T=0$, we expect freeze-out to occur only when the nucleons no longer form interconnected matter. If we use the nucleon radius $r_{n} \simeq 0.8 \mathrm{fm}$ for $r_{h}$, Eq. (44) leads to $n_{f} \simeq 0.16 \mathrm{fm}^{-3}$ as the freeze-out density, and this is in good approximation the density of normal nuclear matter. The correspondence between hadron percolation and freeze-out thus works well at $T=0$. It defines nuclear matter as the most dilute system of nucleons which still forms connected matter.

Can we also relate the other extreme, freeze-out and deconfinement at $\mu=0$, to percolation? Eq. (5) determines the density of hadrons for which the vacuum disappears as a connected medium. From the arguments given above, we can consider the interacting hadron system at $\mu=0$ as an ideal resonance gas. Hence it seems natural to use the vacuum percolation condition

$$
n(T, \mu=0)=\frac{1.24}{(4 \pi / 3) r_{h}^{3}},
$$

for the ideal resonance gas density $n(T, \mu=0)$ to determine both freeze-out and deconfinement. To obtain $n(T, \mu)$ and other necessary observables of such a system, we briefly recall the essentials of the ideal resonance gas model. 
In thermodynamics, a closed system of fixed energy $E$, volume $V$, and number $N_{i}$ of particles of type $i$ is described as microcanonical ensemble. Bringing this system in contact with a heat bath leads to the canonical ensemble, having an average energy $\langle E\rangle=\operatorname{tr}(\rho H)$, while $V$ and $N_{i}$ are conserved exactly. If we further also let the particle numbers $N_{i}$ fluctuate with only their averages conserved, we obtain the grand canonical ensemble. For this, we have

$$
Z_{G}=\operatorname{tr} e^{-\beta\left(H-\mu_{i} N_{i}\right)}=\prod_{i} \sum_{N_{i}} \lambda_{i}^{N_{i}} Z_{N_{i}}
$$

where $\lambda_{i}=e^{\beta \mu_{i}}$ is the fugacity of particle species $i$ and $Z_{N_{i}}$ the corresponding canonical partition function. The chemical potentials $\mu_{i}$ assure particle number conservation in an average sense. Changing the summation over discrete quantum states to phase space integration, we obtain the grand canonical partition in the form

$$
\ln Z_{G}=\sum_{i} g_{i} \frac{V}{2 \pi^{2}} \int_{0}^{\infty} d p p^{2} \ln \left[1+\eta_{i} \lambda_{i} e^{-\beta p_{i}^{0}}\right]^{\eta_{i}}
$$

where $p_{i}^{0}=\sqrt{p^{2}+m_{i}^{2}}$, with $m_{i}$ for the mass of the particle species $i, g_{i}$ counts the spin/isospin degeneracy, and $\eta_{i}$ is the statistics factor, with $\eta_{i}=1$ for fermions and $\eta_{i}=-1$ for bosons.

In a relativistic gas, in which the particle numbers are generally not conserved, the chemical potentials are associated to the conserved baryon, charge and strangeness quantum numbers $B, Q$ and $S$. The abundance of strangeness is complicated by the larger strange quark mass, which could prevent that strange quarks are produced as abundantly as the lighter non-strange quarks. In order to allow for a resulting reduced strangeness production, the parameter $\gamma_{s}\left(\gamma_{s} \leq 1\right)$ was introduced [10]. For $\gamma_{s}=1$, no suppression occurs, while for $\gamma_{s}<1$, fewer strange quarks appear, with a corresponding suppression of strange particle production. A factor $\gamma_{s}$ is associated to each strange quark, so that for hadrons containing $f_{s}$ strange quarks, there is an additional multiplier $\gamma_{s}^{f_{s}}$ in the fugacity. We thus have

$$
\lambda_{i}=\gamma_{s}^{f_{s}} \lambda_{B}^{B_{i}} \lambda_{Q}^{Q_{i}} \lambda_{S}^{S_{i}}
$$

where $B_{i}, Q_{i}$ and $S_{i}$ are the quantum numbers for individual particle species. Then the particle numbers are given by

$$
\left\langle N_{i}\right\rangle=g_{i} \frac{V}{2 \pi^{2}} \int_{0}^{\infty} d p p^{2}\left[\left(\gamma_{s}^{f_{s}} \lambda_{B}^{B_{i}} \lambda_{Q}^{Q_{i}} \lambda_{S}^{S_{i}}\right)^{-1} e^{\beta p_{i}^{0}}+\eta_{i}\right]^{-1}
$$

and the overall density of hadronic constituents by

$$
n(T, \mu)=\frac{\sum_{i}\left\langle N_{i}\right\rangle}{V} .
$$

The average net baryon number, i.e., the number of baryons minus that of antibaryons, is the sum over the particle numbers weighted by the baryon quantum number,

$$
\left\langle N_{B}\right\rangle=\sum_{i} B_{i}\left\langle N_{i}\right\rangle
$$


and the corresponding baryon density is

$$
n_{B}(T, \mu)=\frac{\left\langle N_{B}\right\rangle}{V}
$$

From

$$
\langle E\rangle=\sum_{i} g_{i} \frac{V}{2 \pi^{2}} \int_{0}^{\infty} d p p^{2} p_{i}^{0}\left[\left(\gamma_{s}^{f_{s}} \lambda_{B}^{B_{i}} \lambda_{Q}^{Q_{i}} \lambda_{S}^{S_{i}}\right)^{-1} e^{\beta p_{i}^{0}}+\eta_{i}\right]^{-1}
$$

we obtain with

$$
\epsilon=\frac{\langle E\rangle}{V}
$$

a similar relation for the energy density.

For $\mu=0$, we now use Eq. (11) in the percolation condition (5), including all observed resonances up to mass $2.5 \mathrm{GeV}$, with the nucleon radius $r_{h}=0.8 \mathrm{fm}$ for baryons; for mesons, we use a value smaller by a factor $(2 / 3)^{3 / 4} \simeq 1.1$, as suggested by bag model arguments. This determines the threshold temperature for confinement and (species abundance) freeze-out at vanishing baryon-density, giving $T_{c}=T_{f} \simeq 167 \mathrm{MeV}$ for $\gamma_{s}=1$ and $175 \mathrm{MeV}$ for $\gamma_{s}=0.5$.

We have thus specified $T_{f}=T_{c}$ at $\mu=0$ through vacuum percolation and $\mu_{f}$ at $T=0$ through nucleon percolation.

To define a freeze-out curve in the whole $T-\mu$ plane, we have to combine the resonance gas aspects at low baryon density with the baryon repulsion nature at high baryon density. If we move from $\mu=0$ to finite $\mu$, the resulting medium is no longer an ideal gas, since now baryon interactions are present, which are not accountable in terms of resonances. To reach freeze-out, the system has to expand and cool off enough to stop these non-resonant baryon contributions. It is thus clear that for $\mu \neq 0$, freeze-out will occur for $T_{f}<T_{c}$, below deconfinement. In particular, as is seen from Fig. 2, when we reach $\mu=\mu_{f}$, the interacting hadronic medium formed at the confinement point still contains mesons; but these have disappeared when the medium has cooled off enough to freeze out all dynamical baryon repulsion, leaving cold nuclear matter of standard density.

Given the overall hadron density $n(T, \mu)$ and the net baryon number density $n_{B}(T, \mu)$ through Eq'ns. (11) and (13), respectively, we can specify a freeze-out temperature showing the behaviour just outlined. We assume that the sector of vanishing baryon density freezes out according to the resonance gas approximation and by vacuum percolation, that of finite baryon number according to baryon percolation, to obtain

$$
n(T, \mu)=\frac{1.24}{V_{h}}\left[1-\frac{n_{B}(T, \mu)}{n(T, \mu)}\right]+\frac{0.34}{V_{h}}\left[\frac{n_{B}(T, \mu)}{n(T, \mu)}\right]
$$

as the defining equation for the freeze-out curve. It is clear that when $\mu=0$, we recover condition (5), while in the limit of a cold nucleon gas, with $n / n_{B}=1$, we get back Eq. (41). Moreover, it determines freeze-out fully in terms of geometric clustering based on the intrinsic hadronic scale. The model contains no adjustable parameters, with the exception of a possible $\gamma_{s}<1$. In principle, $\gamma_{s}$ could depend on the initial collider energy or on $\mu$. However, variations of $\gamma_{s}$ have only little effect on the resulting curve, as already noted 
for the limiting case of $\mu=0$, and this effect decreases further for higher $\mu$. Therefore we will perform our calculations for fixed $\gamma_{s}=0.5$.

We now turn to a comparison of our results to the relevant data. Starting with data for freeze-out temperatures at $\mu=0$, we show in Fig. 3 a recent compilation of data from elementary hadron collisions [4]; it is seen that the range $T \simeq 170 \pm 5 \mathrm{MeV}$ obtained from the vacuum percolation condition (4) for $0.5 \leq \gamma_{s} \leq 1$ is in good agreement with the data.

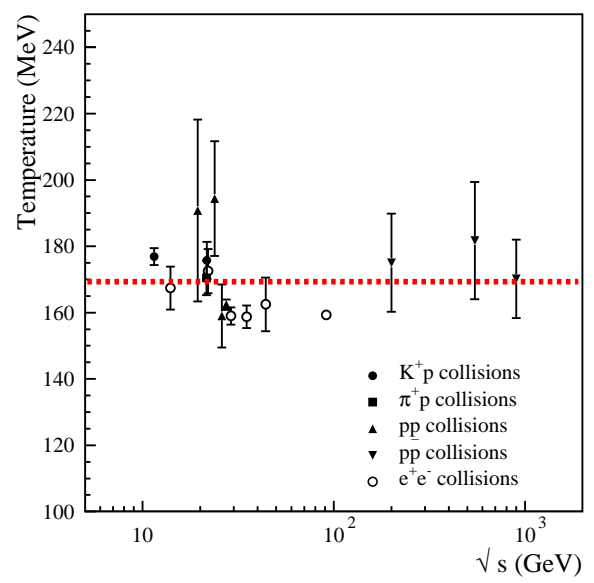

Figure 3: Freeze-out temperature in elementary hadron collisions, from 4].

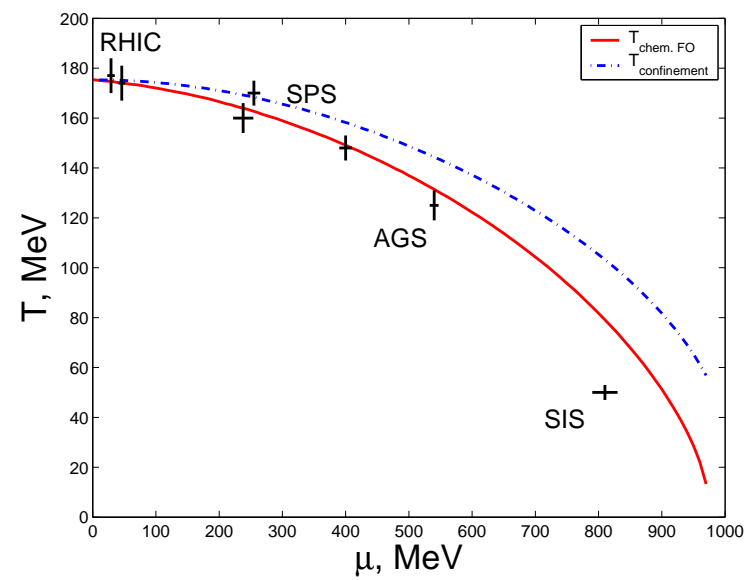

Figure 4: Freeze-out in heavy ion collisions, from [11.

Coming to heavy ion collisions, we show in Fig. 4 results from the four different facilities. Also here the agreement is seen to be very good, with clear deviations only for the lowest energy data from SIS. While we have no explanation for this, it should be noted that at such low energies, secondary hadron production is a rather rare process, making a freeze-out determination from species abundances more difficult. In particular, the number of nucleons is now fixed by the mass of the colliding nuclei, not by any thermal parameter. It is rather surprising that the data fall below the expected curve. Our 
assumption of an ideal nucleon gas at high baryon density could well be an oversimplification; but we saw above that further dynamical repulsion, beyond the effect due to the Fermi statistics of the nucleons, in fact leads to a freeze-out at higher $\mu$. One might thus wonder whether threshold effects at SIS energies could lead to an effective reduction of the freeze-out temperature.

Finally, we compare our freeze-out condition with that obtained from $\langle E\rangle /<$ $N>=1 \mathrm{GeV}$ [7]. In Fig. [5] we show both forms together with the data. While the two models never differ by more than $10 \%$, the form of [7, in contrast to ours, provides good agreement also for the SIS point. On the other hand, $\langle E\rangle /\langle N\rangle=1 \mathrm{GeV}$ leads to a rather sudden change of behaviour for $\mu \geq 0.85 \mathrm{GeV}$, which puts nuclear matter below the freeze-out point in $\mu$.

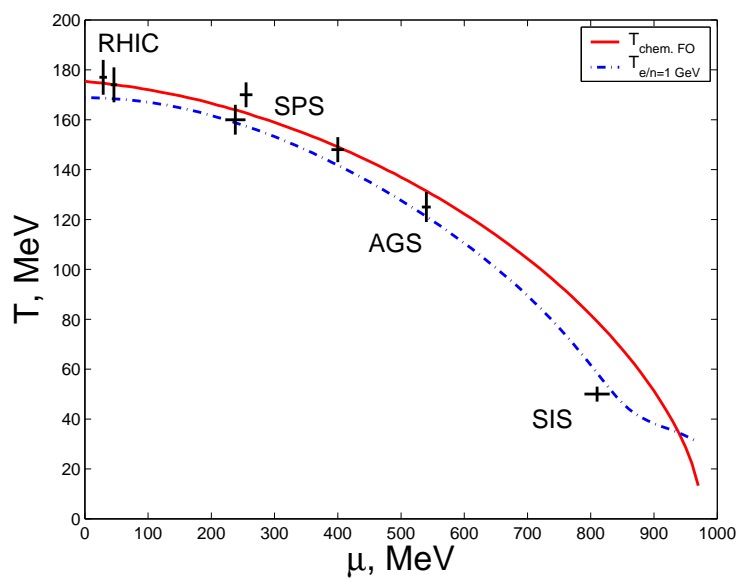

Figure 5: Freeze-out from percolation and from $\langle E\rangle /\langle N\rangle=1 \mathrm{GeV}$.

\section{Acknowledgments}

We thank K. Redlich (Bielefeld and Wroclaw) for helpful discussions and J. Manninen (Oulu) for valuable assistance with programing.

\section{References}

[1] E. Beth and G. E. Uhlenbeck, Physica 4 (1937) 915.

[2] R. Hagedorn, Nuovo Cim. Suppl. 3 (1965) 147;

Nuovo Cim. 56A (1968) 1027.

[3] See e.g., E. DelGiudice et al., Annals Phys. 70 (1972) 378.

[4] F. Becattini, Z. Phys. C 69 (1996) 485;

F. Becattini and U. Heinz, Z. Phys. C 76 (1997) 269;

F. Becattini, hep-ph/0206203

[5] See e.g., F. Karsch, Lect. Notes Phys. 583 (2002) 209 hep-lat/0106019. 
[6] J. Cleymans et al., Z. Phys. C33 (1986) 151.

[7] J. Cleymans and K. Redlich, Phys. Rev. Lett. 81 (1998) 5284.

[8] See e.g., M. S. Isichenko, Rev. Mod. Phys. 64 (1992) 961.

[9] G. Baym, Physica 96A (1979) 131;

T. Çelik, F. Karsch and H. Satz, Phys. Lett. 97B (1980) 128;

H. Satz, Nucl. Phys. A642 (1998) 130c.

[10] J. Rafelski, Phys. Lett. B262 (1991) 333.

[11] For a recent compilation, see e.g. P. Braun-Munzinger et al., nucl-th/0303036. 